\title{
Epothilone KOS-1584
}

National Cancer Institute

\section{Source}

National Cancer Institute. Epothilone KOS-1584. NCI Thesaurus. Code C77892.

A second-generation epothilone with potential antineoplastic activity. Epothilone KOS1584 binds to tubulin and induces microtubule polymerization and stabilizes microtubules against depolymerization, which may result in the inhibition of cell division, the induction of G2/M arrest, and apoptosis. Compared to first-generation epothilones, this agent exhibits greater safety and efficacy with an enhanced pharmaceutical profile, including enhanced water solubility and tumor penetration, and reduced CNS exposure. In addition, epothilone KOS-1584 is a poor substrate for the P-glycoprotein (P-gp) drug efflux pump. 